\title{
Chimiothérapie anticancéreuse et développement dentaire
}

\section{Antineoplastic chemotherapy and dental development}

\author{
LAURENT NAWROCKI*, PHILIPPE LIBERSA*, FABIEN PORÉE**, ANNE LAMBILLIOTTE***, \\ FRANÇOISE MAZINGUE ${ }^{\star \star *}$, BRIGITTE NELKEN ${ }^{\star \star \star}$, JEAN-CLAUDE LIBERSA*.
}

\section{RÉSUMÉ}

Les progrès du traitement des cancers de l'enfant posent le problème des séquelles dues aux thérapeutiques appliquées pendant l'odontogenèse. Si les effets iatrogènes des radiations ionisantes sont bien analysés, les répercussions des seuls antimitotiques sur la dentition sont moins connues. C'est pourquoi, afin d'éviter les biais liés à des observations rétrospectives, 13 enfants souffrant en majorité d'une leucémie aiguë lymphoblastique et tous traités par le même protocole chimiothérapique ont été suivis prospectivement ainsi que 11 enfants témoins. L'influence des antimitotiques sur la dentition est abordée sous deux angles : la mesure de la croissance de la deuxième molaire mandibulaire droite, dent choisie en référence, pendant les différentes phases du protocole chimiothérapique puis en rémission et la surveillance de l'apparition d'anomalies dentaires. Le support est l'utilisation d'orthopantomogrammes tirés à intervalles réguliers après étude du grandissement induit par le générateur.

Les résultats convergent vers un ralentissement de la vitesse de minéralisation coronaire de la 47 par la chimiothérapie d'induction-consolidation alors que le rythme radiculaire semble insensible à toutes les phases de chimiothérapie.

La non prise en compte possible pour le moment d'éventuels défauts structurels amélo-dentinaires sousestime certainement l'importance des troubles. Cependant déjà, 8 enfants sur 13 ont développé des anomalies dentaires corrélées à la période d'administration des antimitotiques. Les troubles les plus récurrents sont des racines dentaires grêles et/ou courtes (5 enfants) et les agénésies (3 enfants). Quelque soit l'anomalie, la dent la plus fréquemment touchée est la seconde prémolaire. Les atteintes les plus importantes (agénésies, microdonties) concernent les enfants traités les plus jeunes. (Med Buccale Chir Buccale 2003; 9: 7-20).

mots clés : Développement dentaire, Chimiothérapie

\section{$\underline{\text { SUMMARY }}$}

Since the incidence of chemotherapy on developing dentition is poorly documented, 13 children affected by an acute lymphoblastic leukemia and treated by polychemotherapy were assessed versus 11 controls. Measurements of tooth growth as well as dental abnormalities were evaluated. Control X-rays have also been performed before and after unduction-consolidation and 6 months after remission. Significant differences regarding the rythms of the crown mineralization between periods with and without induction-consolidation chemotherapy were found. 8 of 13 children showed altered roots (5 children) and hypodontia (8 children) correlated with the anti-leukemia treatment. The second premolar was the most affected tooth. Furthermore, the effects were more important when the chemotherapy was administrated before the age of 5. However, histological studies are required in order to investigate the effect of chemotherapy on dental structures such as enamel. (Med Buccale Chir Buccale 2003; 9: 7-20).

key words : Tooth development, Chemotherapy

\footnotetext{
* Service d'Odontologie, CHRU Lille, France

** Département de Mathématiques et Statistiques, ESTACA Levallois-Perret, France

*** Département d'Onco-Hématologie Pédiatrique, Hôpital Jeanne de Flandre, CHRU Lille, France.
}

Demande de tirés à part :

Laurent Nawrocki 6 rue du curé Saint-Etienne 59800 Lille France. 
médecine

buccale

chirurgie

buccale

VOL. $9, \mathrm{~N}^{\circ} 1$ 2003

page 8
Si les répercussions de la radiothérapie sur le développement dentaire ont été bien analysées par Dechaume et coll. [1], celles dues à la chimiothérapie anticancéreuse sont moins connues. Dans une grande majorité des cas publiés [2-5], l'application de thérapeutiques mixtes, radio/chimiothérapies, rend délicate toute interprétation relative aux conséquences de la seule chimiothérapie sur la dentition. Les progrès considérables effectués depuis vingt ans dans le traitement des cancers de l'enfant ont permis la guérison d'un nombre croissant d'enfants, mais de possibles séquelles des antimitotiques sur la dentition ont été signalées. C'est ainsi qu'en 1984, Jaffe et coll. [6], Kenrad ${ }^{[7]}$ et Welbury et coll. [8] ont présenté des anomalies dentaires (microdonties, hypodonties, hypoplasies amélaires) chez des enfants en longue rémission d'un cancer. En l'absence de radiothérapie et s'agissant de cancers situés hors de la tête et du cou, seule la chimiothérapie a pu sembler en cause.

Depuis cette date, une série de publications majoritairement descriptives et rétrospectives, ont renforcé cette hypothèse : Rosenberg et coll. [9], Maguire et coll. [10], Purdell-Lewis et coll. [4], Nunn et coll. [11] ont trouvé de façon statistiquement significative, des anomalies (agénésies, anomalies radiculaires, hypoplasies) plus nombreuses dans les groupes d'enfants antérieurement traités par chimiothérapie par rapport aux groupes d'enfants témoins.

Salagnac et coll. [12] ont examiné 71 radiographies panoramiques d'enfants ayant souffert de tumeurs diverses. Leurs principales conclusions sont les suivantes :

- quel que soit le sexe, les atteintes les plus sévères concernent les enfants traités les plus jeunes ;

- les dents les plus fréquemment touchées sont à égalité les secondes prémolaires et deuxièmes molaires puis, les premières prémolaires et molaires supérieures ;

- les atteintes (coronaires / radiculaires) dépendent du stade d'édification dentaire au moment du traitement chimiothérapique ;

- tous les sujets ne présentent pas le même degré d'atteinte, cela tient peut-être à une réponse individuelle aux antimitotiques et à une sensibilité des améloblastes et des odontoblastes à ces médicaments pendant une période très précise.

Sonis et coll. [13] en comparant l'état dentaire d'enfants ayant souffert d'une leucémie aiguë lymphoblastique ont montré que comparativement aux enfants ayant reçu une radiothérapie, ceux traités uniquement par chimiothérapie présentaient également des anomalies du développement essentiellement radiculaires. Ils ont établi à la suite des premiers travaux de Dahllöf et coll. [14] une classification de la sévérité des atteintes sous forme de scores.

Cependant ces travaux descriptifs sont critiquables. En effet, ils sont basés pour la plupart sur l'analyse a posteriori d'une seule radiographie, ce qui ne permet pas de connaître la situation initiale et donc de se prononcer de façon certaine, notamment en cas d'agénésie ou de microdontie. De plus, quelques publications $[4,11]$ ne tiennent pas compte de la radiothérapie crânienne préventive des récidives leucémiques à partir des sanctuaires tumoraux méningés (responsabilité démontrée par Dahllöf et coll. [1415]), rendant les conclusions portées peu fiables. Cependant, les expérimentations animales (rats/hamsters) semblent établir la responsabilité des antimitotiques pour les anomalies dentaires rencontrées [16-19]. Face aux inconvénients des méthodes rétrospectives, l'approche prospective assure une connaissance fiable des traitements reçus et constate l'état initial. A partir de radiographies successives, cette étude se propose d'évaluer l'influence de la chimiothérapie sur le rythme de la minéralisation dentaire et devrait permettre de se prononcer sur d'éventuelles anomalies dentaires.

\section{PATIENTS ET METHODES}

Les objectifs suivants ont été définis :

- il s'agit de suivre la dentition d'enfants malades et témoins sur une longue période ;

- l'observation nécessite des radiographies tirées régulièrement à partir du diagnostic tumoral ; - le traitement chimiothérapique doit être identique chez tous les enfants ;

- une bonne répartition des âges de recrutement est nécessaire, afin de visualiser les éven- 
tuels effets des antimitotiques aux différents stades de formation des dents ;

- aucune radiothérapie ne doit concerner la tête ou le cou ;

Après l'avis favorable du CCPPRB, les enfants souffrant d'une Leucémie Aiguë Lymphoblastique ( $L A L)$ de risque standard ou d'un Lymphome Malin Non Hodgkinien (LMNH) de type non $B$ ont été retenus. En effet ces deux pathologies reçoivent le même protocole chimiothérapique $\mathrm{n}^{\circ} 58881$ de l'EORTC (European Organization for Research and Treatment in Cancer), la radiothérapie n'étant employée que pour des formes plus sévères (irradiation corps entier avant greffe de moelle osseuse, irradiation méningée préventive des récidives) [20].

Le traitement de la forme standard comporte une phase d'induction-consolidation (IC) d'une durée de 6 mois puis une phase d'entretien $(E)$ de 18 mois. La phase IC se décompose en :

- préphase (à base de prednisolone, méthotrexate intrathécal [IT]),

- induction (prednisolone, vincristine, daunorubicine, méthotrexate IT, asparaginase),

- consolidation (cyclophosphamide, méthotrexate IT, purinéthol, aracytine),

- traitement intermédiaire (purinéthol, méthotrexate, méthotrexate IT, acide folinique),

- réinduction-consolidation (dexaméthasone, vincristine, adriamycine, asparaginase, aracytine, 6-thioguanine, cyclophosphamide, méthotrexate IT).

La phase E comporte (formes orales) du purinéthol et du méthotrexate. L'intensité moindre, le nombre de drogues et le dosage plus faibles caractérisent cette phase par rapport à la précédente. Les témoins sont des enfants consultant le Service d'Odontologie pour soins dentaires.

Aucune technique radiographique n'apparaît en complète adéquation avec le double objectif poursuivi, c'est à dire, la précision pour la réalisation de mesures et la vision globale nécessaire au dépistage d'anomalies. Les techniques intra-orales n'ont pas été retenues devant la multiplication des clichés et les difficultés de réalisation chez de très jeunes enfants. Parmi les techniques extra-orales, l'orthopantomogramme (OPT) a été choisi pour sa vision claire de la dentition. Ainsi les OPT des malades et des témoins seront réalisés dans le service d'odontologie avec le même générateur. Cependant, cette technique génère, notamment, un grandissement variable selon les appareils [21]. Celui-ci a été étudié au niveau de la branche horizontale de la mandibule car l'image y est constamment bonne [21-22]. Pour cela chez 11 enfants témoins, deux clichés de nature différente ont été tirés lors d'une même consultation et ce, de deux à cinq reprises suivant les cas. Le premier cliché est un OPT tandis que le second est une radiographie rétro-alvéolaire $(\mathrm{RA})$ de la deuxième molaire mandibulaire droite (47) tirée en technique parallèle long-cône, avec utilisation d'un angulateur. Les RA sont munies d'une grille millimétrée métallique autocollante, avec épaississement centimétrique, ainsi est vérifiée la stabilité de chaque RA puis le grandissement de I'OPT est déterminé en comparant les longueurs de la 47 entre les deux clichés. Les repères de mesure sont pour les deux types de clichés, la longueur totale de la 47 depuis la pointe cuspidienne distale jusqu'à l'extrémité distale coronaire ou radiculaire formée à ce stade.

33 OPT et 33 RA ont été réalisés chez 11 enfants témoins (Tab. 1). La technique rétro-alvéolaire est précise puisque aucun grandissement n'a été constaté sur 33 clichés, le centimètre de la grille se mesurant très exactement sur chaque RA. Le grandissement des 33 OPT varie de 5.88 à $25.00 \%$ en étant assez stable chez un même sujet avec une variation de $+/-4 \%$ sauf pour les témoins $n^{\circ} 4$ et $n^{\circ} 8(+/-9 \%)$.

Afin de déterminer un estimateur efficace du grandissement de l'OPT par rapport à la RA, on utilise la méthode statistique des moindres carrés. Son emploi est légitime car le coefficient de corrélation de la série statistique étudiée est $\approx 0.9921$. Cette méthode consiste à déterminer la pente $(\bullet)$ et l'ordonnée à l'origine (B) de la droite qui minimisent la somme des carrés des écarts résiduels. Une telle droite a pour équation : y $(\mathrm{OPT})=\bullet x(\mathrm{RA})+\mathrm{B}$ où $a=$ tangente q représente la pente de la droite de régression de y en $x$ (Fig.1). C'est un estimateur efficace du grandissement de l'OPT par rapport à la RA. Les données du tableau 1 aboutissent à l'équation suivante: $y=1.0822 x+0.4506$. médecine

buccale chirurgie buccale

VOL. $9, \mathrm{~N}^{\circ} 1$ 2003

page 9 
Tableau 1 : Etude du grandissement radiographique de l'OPT The study of panoramic radiography enlargement

\begin{tabular}{|c|c|c|c|c|}
\hline $\mathbf{N}^{\circ}$ du patient & Date des clichés & $\begin{array}{l}\text { Mesure du } \\
\text { segment sur } \\
\text { la RA (mm) }\end{array}$ & $\begin{array}{l}\text { Mesure du } \\
\text { segment sur } \\
\text { I'OPT (mm) }\end{array}$ & $\begin{array}{l}\text { Coefficient de } \\
\text { grandissement }\end{array}$ \\
\hline 1 & 22-09-1995 & 06.00 & 07.00 & 1.1667 \\
\hline 1 & 13-03-1996 & 06.50 & 07.50 & 1.1538 \\
\hline 1 & 03-07-1996 & 07.00 & 08.00 & 1.1429 \\
\hline 2 & 09-10-1995 & 06.25 & 07.50 & 1.2000 \\
\hline 2 & 27-03-1996 & 07.00 & 08.50 & 1.2143 \\
\hline 2 & 19-08-1996 & 07.50 & 09.00 & 1.2000 \\
\hline 3 & 19-04-1995 & 07.50 & 08.00 & 1.0667 \\
\hline 3 & $31-01-1996$ & 08.00 & 08.50 & 1.0625 \\
\hline 3 & 25-09-1996 & 08.50 & 09.00 & 1.0588 \\
\hline 4 & $15-05-1995$ & 03.00 & 03.50 & 1.1667 \\
\hline 4 & $18-10-1995$ & 04.00 & 05.00 & 1.2500 \\
\hline 4 & $16-07-1996$ & 05.50 & 06.50 & 1.1818 \\
\hline 5 & $15-12-1994$ & 09.50 & 10.50 & 1.1053 \\
\hline 5 & $16-04-1995$ & 10.50 & 11.50 & 1.0952 \\
\hline 5 & 06-03-1996 & 11.00 & 12.00 & 1.0909 \\
\hline 5 & 26-06-1996 & 11.50 & 12.50 & 1.0870 \\
\hline 5 & $22-11-1996$ & 13.00 & 14.00 & 1.0769 \\
\hline 6 & $05-10-1995$ & 11.00 & 12.00 & 1.0909 \\
\hline 6 & 13-03-1996 & 11.50 & 13.00 & 1.1304 \\
\hline 6 & 03-07-1996 & 12.00 & 13.50 & 1.1250 \\
\hline 7 & 22-01-1996 & 07.00 & 08.00 & 1.1429 \\
\hline 7 & 24-04-1996 & 08.00 & 09.00 & 1.1250 \\
\hline 7 & $18-09-1996$ & 09.50 & 10.50 & 1.1053 \\
\hline 8 & 09-05-1995 & 09.00 & 10.00 & 1.1111 \\
\hline 8 & $25-10-1995$ & 09.50 & 11.00 & 1.1579 \\
\hline 8 & 10-04-1996 & 10.00 & 12.00 & 1.2000 \\
\hline 9 & $11-10-1995$ & 09.00 & 10.50 & 1.1667 \\
\hline 9 & 09-04-1996 & 10.00 & 11.50 & 1.1500 \\
\hline 9 & 26-07-1996 & 10.50 & 12.00 & 1.1429 \\
\hline 10 & $15-01-1996$ & 09.50 & 11.00 & 1.1579 \\
\hline 10 & $12-06-1996$ & 11.50 & 13.00 & 1.1304 \\
\hline 11 & 07-11-1995 & 12.50 & 14.50 & 1.1600 \\
\hline 11 & 19-06-1996 & 14.00 & 16.00 & 1.1429 \\
\hline
\end{tabular}

\section{Principes d'étude à partir de la dent de référence}

La 47 a été choisie en raison de la longue durée de son édification : début à l'âge de 2-3 ans, fin de la couronne vers 8 ans, éruption vers 13 ans (apexogenèse exclue) [23-24]. Ceci correspond aux âges prévisibles de recrutement des patients d'autant que la réalisation d'OPT serait difficile chez un enfant âgé de moins de 3-4 ans. Elle appartient au secteur le moins déformé de I'OPT [21-22] dont le grandissement vient d'être étudié, n'est pas une dent terminale et figurerait parmi les dents les plus atteintes par les antimitotiques [12].

Blocquel et coll. ont montré que l'édification coronaire et l'édification radiculaire ont lieu sur des rythmes distincts mais proches d'un tiers de couronne ou de racine par an [23]. Ceci conduit à constituer deux sous-groupes au sein de chaque groupe et à utiliser des repères différents de l'étude du grandissement. Pour les enfants en phase d'édification coronaire de la 47 (sous-groupe C), la mesure s'effectue entre le fond du sillon occlusal médian vestibulo-lingual et la limite de la zone minéralisée dans sa défini- 


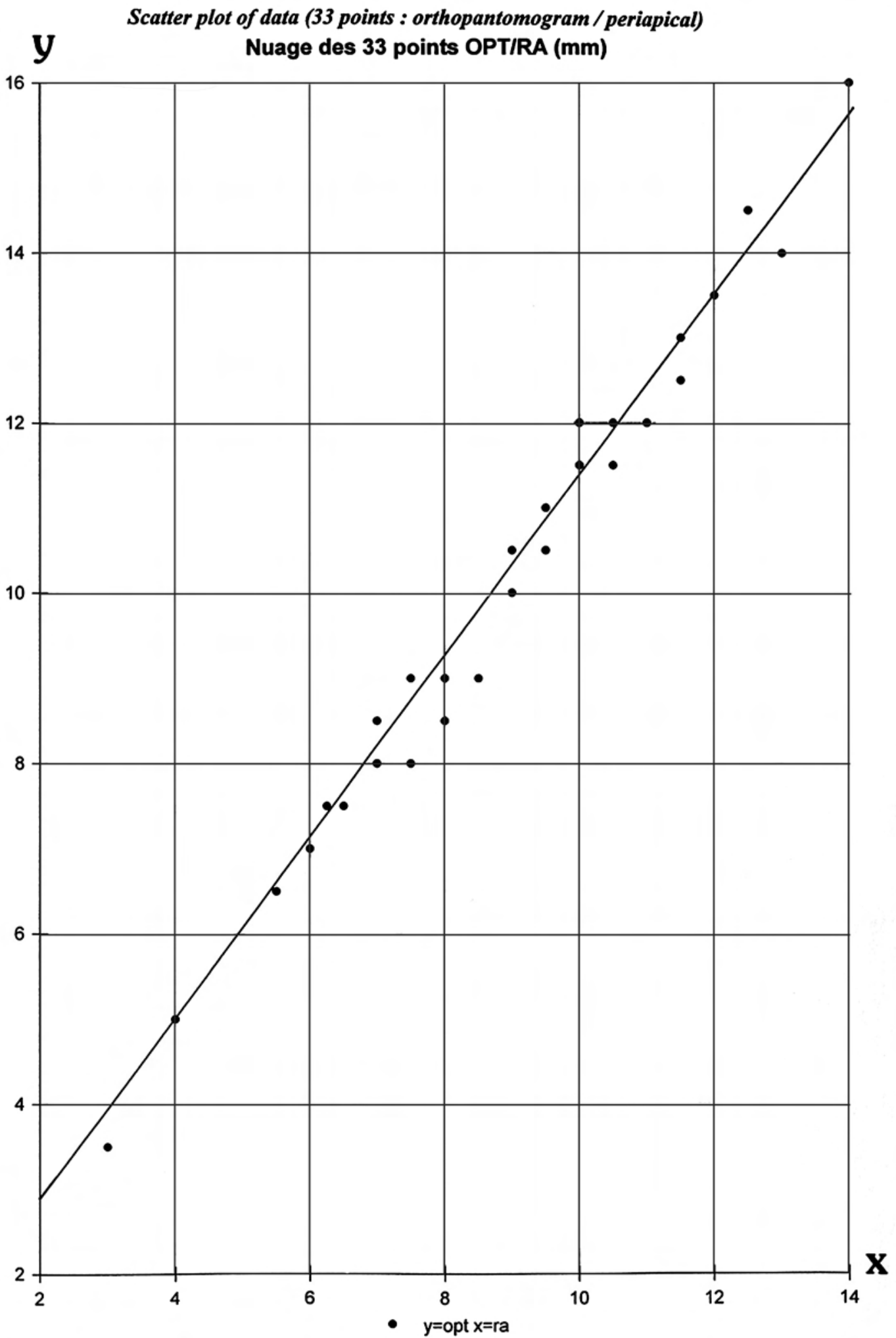

médecine

buccale

chirurgie

buccale

VOL. $9, \mathrm{~N}^{\circ} 1$

2003

page 11

Figure 1 : Droite des moindres carrés

Graph of the least square line 


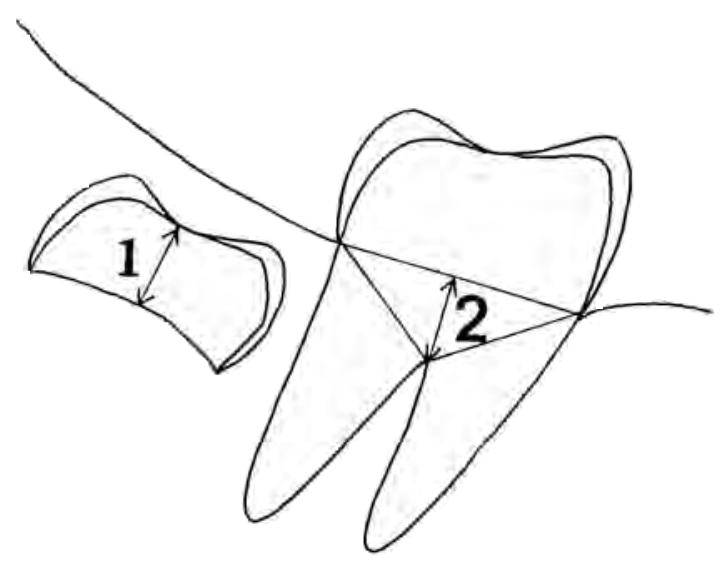

Figure 2 : Mesure coronaire (1) et segment-test Measurement of the crown (1) and segment test (2)

tion la plus étroite (sommet de la concavité)

médecine buccale chirurgie buccale

VOL. $9, \mathrm{~N}^{\circ} 1$ 2003

page 12
(Fig. 2). Pour ceux en phase d'édification radiculaire de la 47 (sous-groupe R), apexogenèse exclue, la mesure s'effectue sur la racine distale depuis la jonction amélo-radiculaire jusqu'à l'extrémité formée, dans sa définition la plus courte en cas de terminaison en biseau (Fig. 3).

Pour le groupe malades (M), compte tenu de la séparation du traitement chimiothérapique en deux phases distinctes, induction-consolidation et entretien [20], les radiographies sont tirées ainsi : le cliché $n^{\circ} 1$ à l'entrée, $n^{\circ} 2$ à la fin de l'induction-consolidation, $\mathrm{n}^{\circ} 3$ à la fin de l'entretien, $\mathrm{n}^{\circ} 4$ en rémission. Ainsi le temps écoulé entre deux clichés est défini suivant qu'il comporte ou non un traitement antimitotique et si oui avec le type de chimiothérapie IC/E. Pour le groupe témoins $(T)$, à partir du cliché $\mathrm{n}^{\circ} 1$ à l'entrée, un rythme annuel de minéralisation est établi grâce à deux clichés semestriels $n^{\circ} 2$ et $n^{\circ} 3$. Dans tous les cas (étude du grandissement et mesures sur les OPT) une double lecture a été effectuée.

\section{Stabilité du grandissement des OPT, annualisation et significativité des mesures}

Le calcul de l'accroissement coronaire ou radiculaire réel de la 47 nécessite la vérification de la stabilité du grandissement des OPT successifs d'un même patient, aussi la conservation

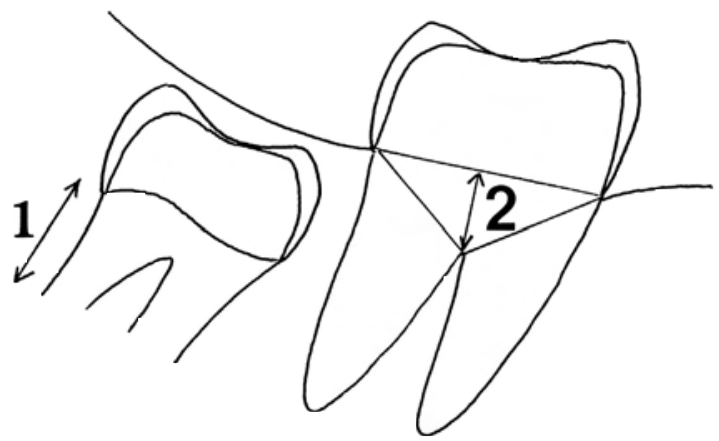

Figure 3 : Mesure radiculaire (1) et segment-test (2) Measurement of the root (1) and segment test (2)

longitudinale des mensurations d'un segmenttest est contrôlée sur les clichés. Ce segment est déterminé sur la 46, la minéralisation y étant achevée dans tous les cas étudiés (Fig. 2 et 3). Afin d'obtenir des accroissements réels comparables, ceux-ci seront annualisés (365 j) en tenant compte des années bissextiles. Certains accroissements seront notés non significatifs (NS) si la dent entre en apexogenèse ou si la dent n'a pas encore débuté sa minéralisation sur le premier cliché, le démarrage de l'édification coronaire ne pouvant être daté.

\section{Calcul des accroissements}

- Malades : suivant l'équation

$y=1.0822 x+0.4506$

$x=\frac{y-0.4506}{1.0822}$

où $\mathrm{y}=$ mesure sur l'OPT et $\mathrm{x}=$ la mesure prédictive sans grandissement sur la RA.

[accroissement réel $=$ différence entre 2 mesures successives vérifiées (et) corrigées].

- Témoins : un calcul propre à chaque patient est réalisé grâce au coefficient individuel du grandissement OPT/RA : [accroissement réel $=$ différence entre 2 mesures corrigées]

- Malades et Témoins :

[accroissement réel annuel $=$ accroissement mesuré corrigé $X 365$ nombre de jours écoulé entre deux clichés 
Tableau 2 : Résultats des deux groupes

Results in control and treated groups

\begin{tabular}{|c|c|c|c|c|c|c|}
\hline $\begin{array}{l}\text { Groupe } \\
(\mathrm{M} / \mathrm{T})\end{array}$ & $\begin{array}{l}\text { Sous-groupe } \\
\text { (C / R) }\end{array}$ & $\begin{array}{l}\mathrm{N}^{\circ} \mathrm{du} \\
\text { patient }\end{array}$ & $\begin{array}{l}\text { Chimiothérapie } \\
\text { (Oui / Non) }\end{array}$ & $\begin{array}{l}\text { Phase de } \\
\text { chimio. } \\
\text { (IC/E) }\end{array}$ & $\begin{array}{l}\text { Anomalie } \\
\text { (Oui / Non) }\end{array}$ & $\begin{array}{c}\text { Accroissement } \\
\text { (mm.an-1) }\end{array}$ \\
\hline$M$ & C & 1 & 0 & $\mathrm{IC}+\mathrm{E}$ & 0 & 1.26 \\
\hline$M$ & C & 1 & $\mathrm{~N}$ & & & 1.30 \\
\hline$M$ & C & 1 & $\mathrm{~N}$ & & & 1.30 \\
\hline$M$ & C & 2 & 0 & IC & 0 & NS \\
\hline$M$ & C & 2 & 0 & $E$ & & 1.31 \\
\hline$M$ & C & 3 & 0 & IC & 0 & 0.97 \\
\hline$M$ & C & 3 & 0 & $E$ & & 0.91 \\
\hline$M$ & C & 3 & $\mathrm{~N}$ & & & 1.52 \\
\hline$M$ & C & 4 & 0 & IC & 0 & 0.89 \\
\hline$M$ & C & 4 & 0 & $E$ & & 1.98 \\
\hline$M$ & C & 4 & $\mathrm{~N}$ & & & 2.20 \\
\hline$M$ & C & 5 & 0 & IC & $\mathrm{N}$ & 1.28 \\
\hline$M$ & C & 5 & 0 & $E$ & & 1.85 \\
\hline$M$ & C & 5 & $\mathrm{~N}$ & & & 1.99 \\
\hline$M$ & C & 6 & 0 & IC & 0 & 0.83 \\
\hline$M$ & C & 6 & 0 & $E$ & & 2.24 \\
\hline$M$ & C & 6 & $\mathrm{~N}$ & & & 2.52 \\
\hline$M$ & C & 7 & 0 & IC & 0 & 0.77 \\
\hline$M$ & C & 7 & 0 & $E$ & & 1.01 \\
\hline$M$ & C & 7 & $\mathrm{~N}$ & & & 1.27 \\
\hline$M$ & C & 8 & 0 & IC & 0 & 0.88 \\
\hline$M$ & C & 8 & 0 & $E$ & & 0.97 \\
\hline$M$ & C & 8 & $\mathrm{~N}$ & & & 1.82 \\
\hline$M$ & C & 9 & 0 & IC & $\mathrm{N}$ & 0.61 \\
\hline$M$ & C & 9 & 0 & $E$ & & 1.26 \\
\hline$M$ & C & 9 & $\mathrm{~N}$ & & & 2.66 \\
\hline$M$ & C & 10 & 0 & IC & 0 & 1.78 \\
\hline$M$ & C & 10 & O & $E$ & & 2.06 \\
\hline$M$ & $\mathrm{R}$ & 1 & O & IC & $\mathrm{N}$ & 0.98 \\
\hline$M$ & $\mathrm{R}$ & 1 & 0 & $\mathrm{E}$ & & 2.72 \\
\hline$M$ & $\mathrm{R}$ & 1 & $\mathrm{~N}$ & & & 2.87 \\
\hline$M$ & $\mathrm{R}$ & 2 & 0 & IC & $\mathrm{N}$ & 0.85 \\
\hline$M$ & $\mathrm{R}$ & 2 & 0 & $E$ & & 1.43 \\
\hline$M$ & $\mathrm{R}$ & 2 & $\mathrm{~N}$ & & & 1.83 \\
\hline$M$ & $\mathrm{R}$ & 3 & 0 & IC & 0 & 1.61 \\
\hline $\mathrm{T}$ & C & 1 & & & & 1.88 \\
\hline $\mathrm{T}$ & C & 1 & & & & 1.56 \\
\hline $\mathrm{T}$ & C & 2 & & & & 1.56 \\
\hline $\mathrm{T}$ & C & 2 & & & & 1.19 \\
\hline $\mathrm{T}$ & C & 3 & & & & 1.21 \\
\hline $\mathrm{T}$ & C & 3 & & & & 2.19 \\
\hline $\mathrm{T}$ & C & 4 & & & & 1.46 \\
\hline $\mathrm{T}$ & C & 4 & & & & 1.88 \\
\hline $\mathrm{T}$ & $\mathrm{R}$ & 1 & & & & 1.43 \\
\hline $\mathrm{T}$ & $\mathrm{R}$ & 1 & & & & 1.04 \\
\hline $\mathrm{T}$ & $\mathrm{R}$ & 1 & & & & 1.54 \\
\hline $\mathrm{T}$ & $\mathrm{R}$ & 1 & & & & 2.37 \\
\hline $\mathrm{T}$ & $\mathrm{R}$ & 2 & & & & 1.71 \\
\hline $\mathrm{T}$ & $\mathrm{R}$ & 2 & & & & 1.50 \\
\hline $\mathrm{T}$ & $\mathrm{R}$ & 3 & & & & 1.79 \\
\hline T & $\mathrm{R}$ & 3 & & & & 2.29 \\
\hline $\mathrm{T}$ & $\mathrm{R}$ & 4 & & & & 0.66 \\
\hline $\mathrm{T}$ & $\mathrm{R}$ & 4 & & & & 0.59 \\
\hline $\mathrm{T}$ & $\mathrm{R}$ & 5 & & & & 1.80 \\
\hline $\mathrm{T}$ & $\mathrm{R}$ & 5 & & & & 1.53 \\
\hline $\mathrm{T}$ & $\mathrm{R}$ & 6 & & & & 2.50 \\
\hline $\mathrm{T}$ & $\mathrm{R}$ & 7 & & & & 1.60 \\
\hline
\end{tabular}

médecine

buccale chirurgie buccale

VOL. $9, \mathrm{~N}^{\circ} 1$ 2003

page 13 
Les comparaisons des rythmes de minéralisation de 47 entres les groupes $M$ et $T$ et entre les périodes et phases de chimiothérapie ont été réalisées avec les test de Kruskal et Wallis et de Wilcoxon. Le seuil de significativité adopté est de 0,05 .

\section{RESULTATS}

Le groupe $M$ est constitué de 13 enfants (sept filles/six garçons), souffrant d'une LAL de risque standard ou d'un LMNH non B et tous traités par le même protocole. Au départ âgés de 2 ans 11 mois à 10 ans 6 mois (moyenne : 5 ans 8 mois), ils ont été suivis pendant des périodes allant jusqu'à 3 ans; 10 sont en phase d'édification coronaire ( $\mathrm{C} 1$ à $\mathrm{C} 10)$ et 3 en phase d'édification radiculaire ( $\mathrm{R} 1$ à $\mathrm{R} 3$ ).

Le groupe $T$ est formé des 11 enfants témoins (sept filles/quatre garçons) ayant aussi participé

médecine buccale chirurgie buccale

VOL. $9, \mathrm{~N}^{\circ} 1$ 2003

page 14 à l'étude du grandissement. Au départ âgés de

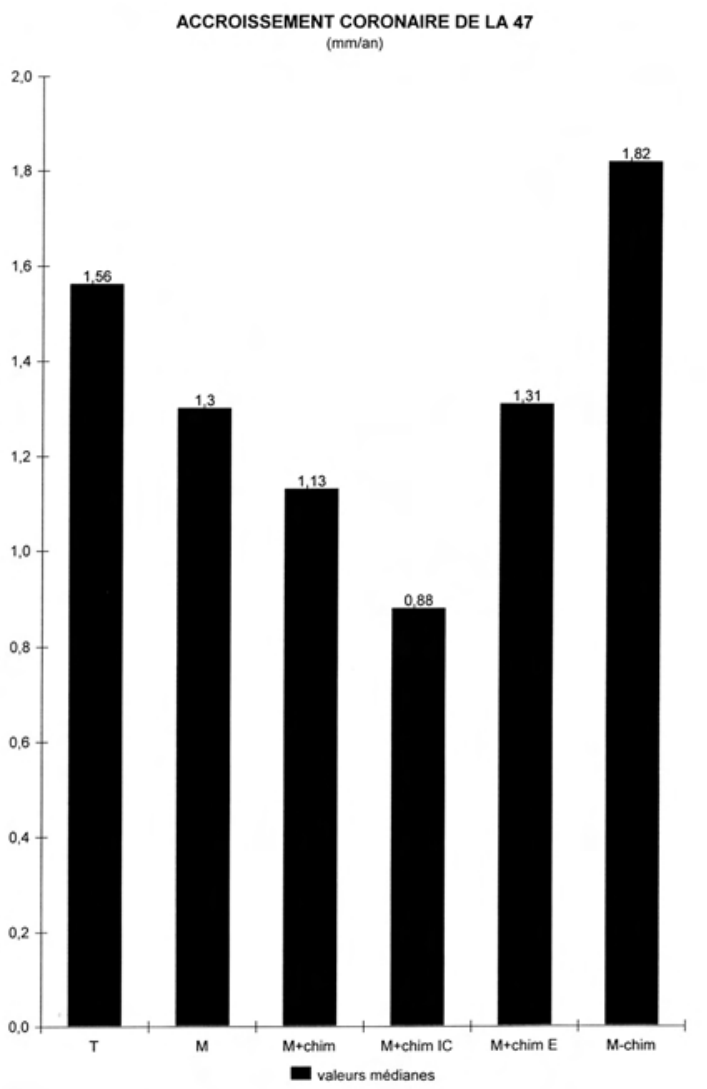

Figure 4 : Accroissement coronaire de la $47\left(\mathrm{~mm}^{-a^{-1}}\right)$ Crown mineralization rythm of the mandibular second right molar (mm.year')
5 ans 8 mois à 10 ans 5 mois (moyenne : 8 ans 1 mois), ils ont été suivis pendant une année. 4 sont en phase d'édification coronaire ( $\mathrm{C} 1$ à $\mathrm{C} 4)$ et 7 en phase d'édification radiculaire (R1 à $R 7)$ (Tab. 2).

Au niveau coronaire, le rythme de minéralisation global des malades (1.3 mm.an-1) est inférieur à celui des témoins (1.56 mm.an-1). Ceci n'est pas retrouvé au niveau radiculaire où les rythmes sont voisins, respectivement 1.61 et $1.57 \mathrm{~mm}^{-a^{-1}}$ (Fig 4 et 5). Pendant la chimiothérapie les rythmes, radiculaire $\left(1.43 \mathrm{~mm}^{-a^{-1}}\right)$ et surtout coronaire (1.13 mm.an $\left.{ }^{-1}\right)$, sont inférieurs à ceux des témoins, respectivement 1.57 et $1.56 \mathrm{~mm}^{-a^{-1}}$.

Les rythmes les plus faibles sont observés en chimiothérapie d'induction-consolidation (IC) : couronne $=0.88 \mathrm{~mm} \cdot \mathrm{an}^{-1}$, racine $=0.98 \mathrm{~mm} \cdot \mathrm{an}^{-1}$. Après la chimiothérapie intensive IC, les rythmes augmentent en chimiothérapie d'entretien $E$, se rapprochent (couronne $=1.31 \mathrm{~mm}^{-a^{-1}}$ ) ou même

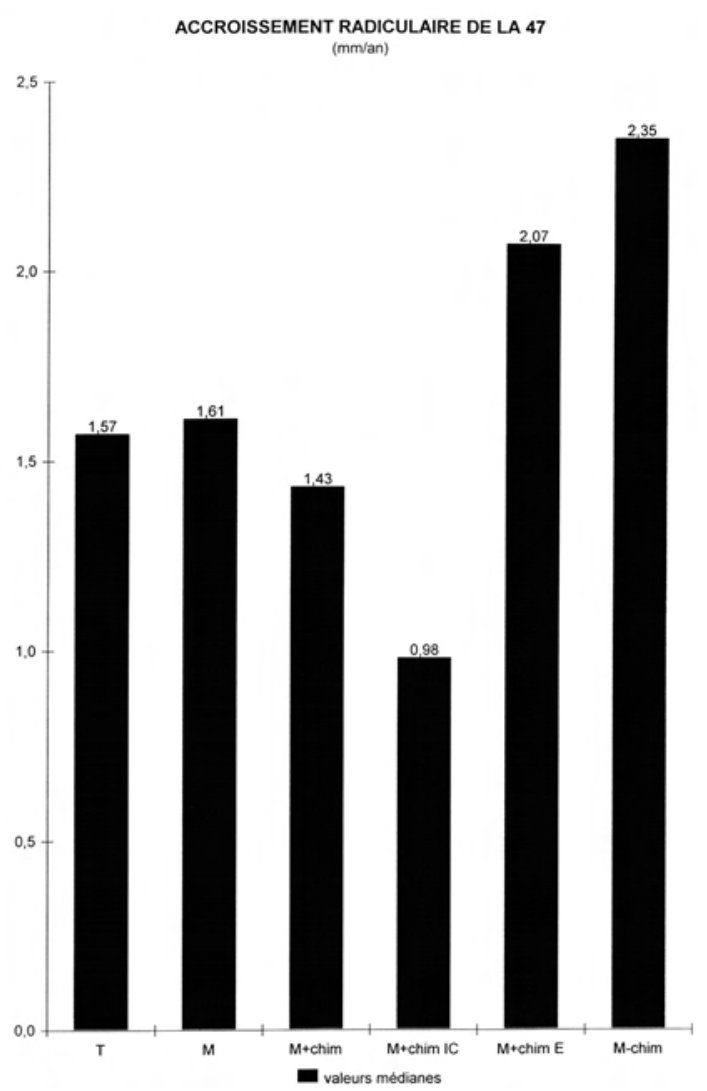

Figure 5 : Accroissement radiculaire de la $47\left(\mathrm{~mm}^{-a n}{ }^{-1}\right)$ Root mineralization rythm of the mandibular second right molar (mm.year') 
dépassent (racine $=2.07 \mathrm{~mm} \cdot \mathrm{an}^{-1}$ ) ceux des témoins. Les rythmes augmentent encore après l'arrêt de la chimiothérapie $E$, c'est à dire en dehors de tout traitement et dépassent ceux des témoins.

\section{Anomalies dentaires}

Les anomalies dentaires du groupe $\mathrm{M}$ touchent 8 patients sur 13 (Tab 3). Pour ceux-ci, la moyenne d'âge d'administration de la chimiothérapie se situe à 4 ans 5 mois. Notons que l'évaluation des anomalies ne tient pas compte d'éventuels défauts amélo-dentinaires. Or pour la plupart des auteurs, ils occupent une place prépondérante dans la hiérarchie des troubles dentaires liés aux drogues anticancéreuses $[8,10,12,25-26]$. II faudra attendre l'érup- tion de l'ensemble des dents permanentes pour l'établir.

Concernant le type d'atteinte, 3 enfants présentent des agénésies multiples. Elles touchent les secondes prémolaires maxillaires et/ou mandibulaires et sont bilatérales (Fig. 6, 7 et 8). Elles représentent ici à titre indicatif $23.08 \%$ des enfants, c'est à dire le triple des statistiques de la population générale [27-29], ce pourcentage est à rapprocher des $19 \%$ de l'étude de Welbury [8]. Pour ces enfants, l'âge moyen de début de la chimiothérapie est de 3 ans 9 mois. Les atteintes radiculaires concernent 5 patients (racine courtes et/ou grêles) soit 38.46 \% à titre indicatif (Fig 9 à 13). Sur ce point les résultats varient de 16 [8] à $50 \%$ [10] suivant les études.

Tableau 3 : Anomalies dentaires des patients des deux groupes

Dental abnormalities in control and treated groups

médecine

buccale

chirurgie

buccale

VOL. $9, \mathrm{~N}^{\circ}$ 2003

page 15

\begin{tabular}{|c|c|c|c|c|c|c|}
\hline Patient & Maladie & $\begin{array}{l}\text { Âge d'adm de la } \\
\text { chimiothérapie }\end{array}$ & $\begin{array}{c}\text { Traitement } \\
\text { Durée de la } \\
\text { chimiothérapie }\end{array}$ & Protocole & Radiothérapie & Anomalies \\
\hline $\mathrm{M}-\mathrm{C} 1$ & LAL & $4 a$ & $2 a$ & EORTC & non & $\begin{array}{c}\text { Racines grêles } \\
16,26\end{array}$ \\
\hline M-C2 & LAL & 2 a 6 m & 1 a $8 m$ & EORTC & non & $\begin{array}{c}\text { Agénésies } \\
15,25,35,45 \\
\text { microdontie } 47\end{array}$ \\
\hline M-C3 & LAL & 5 a $5 \mathrm{~m}$ & $2 a$ & EORTC & non & $\begin{array}{c}\text { Racines grêles } \\
16,26\end{array}$ \\
\hline M-C4 & $L A L$ & 4 a $1 \mathrm{~m}$ & $2 \mathrm{a}$ & EORTC & non & $\begin{array}{c}\text { Agénésies } \\
15,25,35,45\end{array}$ \\
\hline M-C6 & LAL & 4 a $3 \mathrm{~m}$ & $2 a$ & EORTC & non & $\begin{array}{l}\text { Racines grêles } \\
16,26,36,46\end{array}$ \\
\hline $\mathrm{M}-\mathrm{C} 7$ & LAL & 5 a $7 \mathrm{~m}$ & $2 \mathrm{a}$ & EORTC & non & $\begin{array}{c}\text { Racines grêles } \\
16,26,46\end{array}$ \\
\hline M-C8 & LAL & 5 a $6 \mathrm{~m}$ & $2 \mathrm{a}$ & EORTC & non & $\begin{array}{c}\text { Racines courtes } \\
31,41 \\
\text { racines grêles } \\
16,26\end{array}$ \\
\hline M-C10 & LAL & 4 a 8 m & $2 a$ & EOR.C & non & $\begin{array}{l}\text { Agénésies } \\
15,25\end{array}$ \\
\hline T-R7 & $(-)$ & $(-)$ & $(-)$ & $(-)$ & $(-)$ & Agénésie 35 \\
\hline
\end{tabular}

Les patients suivants ne présentent pas d'anomalies (à l'exclusion d'éventuels défauts amélodentinaires) bien qu'ayant suivi le même protocole EORTC d'une durée de 2 ans :
M-C5 (4 a 3 m)
$\mathrm{M}-\mathrm{C9}(4 \text { a } 9 \mathrm{~m})^{*}$
$\mathrm{M}-\mathrm{R} 1(10 \text { a } 3 \mathrm{~m})^{*}$
M-R2 (6 a $9 \mathrm{~m})^{\star}$
M-R3 (8 a 8 m)*

* = âge d'administration de la chimiothérapie 


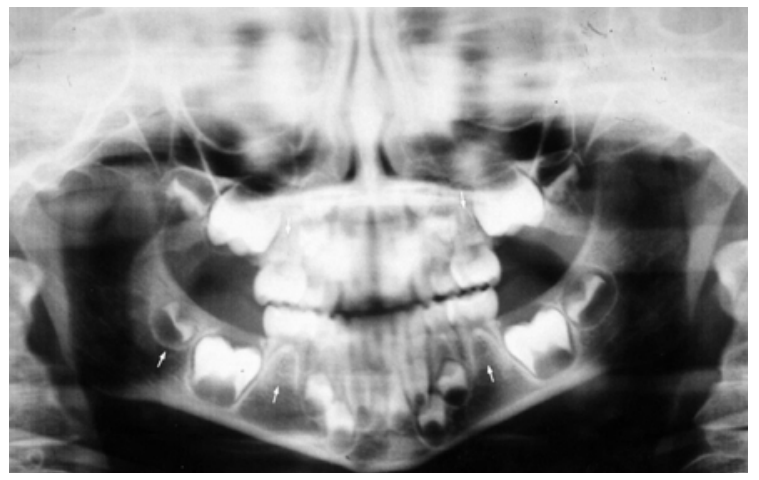

Figure 6 : anomalies dentaires du patient M-C2 : agénésies $(15,25,35,45)$, microdontie (47)

Dental anomalies of the patient M-C2: hypodontia (15, $25,35,45)$, microdontia (47)

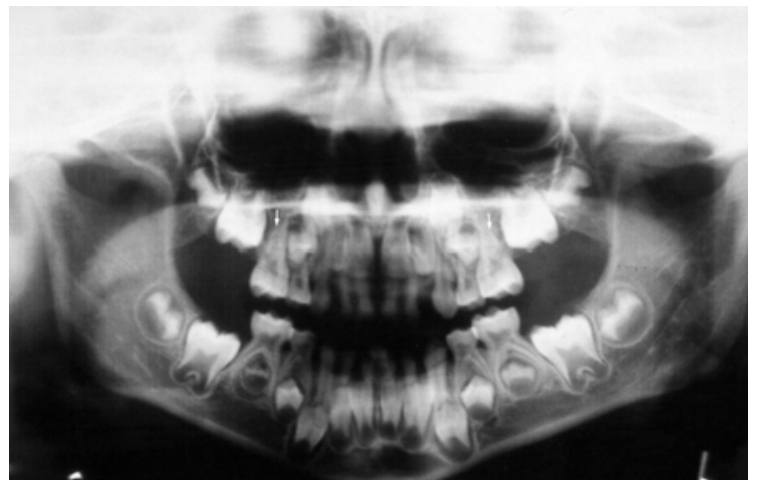

Figure 8 : Anomalies dentaires du patient $\mathrm{M}-\mathrm{C} 10$ : agénésies $(15,25)$

Dental anomalies of the patient M-C10: hypodontia (15, 25)

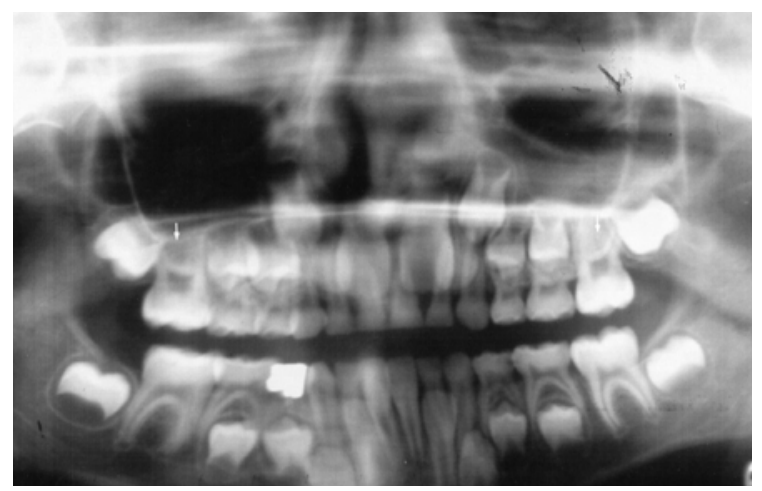

Figure 10 : Anomalies dentaires du patient M-C3 : racines grêles $(16,26)$

Dental anomalies of the patient M-C3: thinned roots $(16,26)$

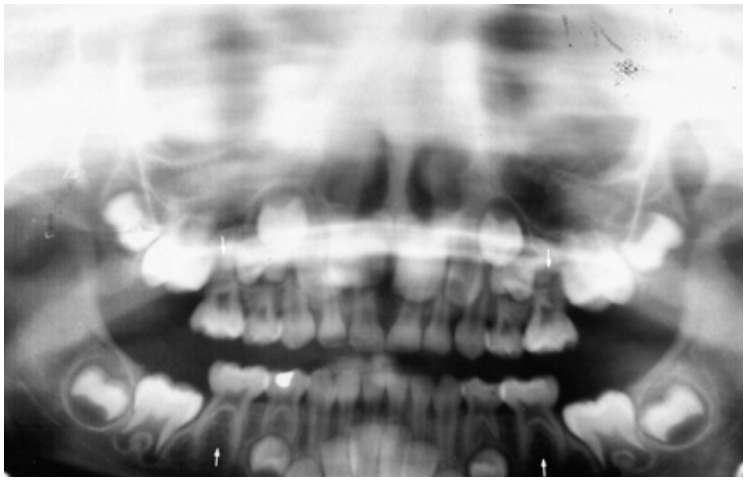

Figure 7 : anomalies dentaires du patient $\mathrm{M}-\mathrm{C} 4$ : agénésies $(15,25,35,45)$

Dental anomalies of the patient M-C4: hypodontia (15, $25,35,45)$

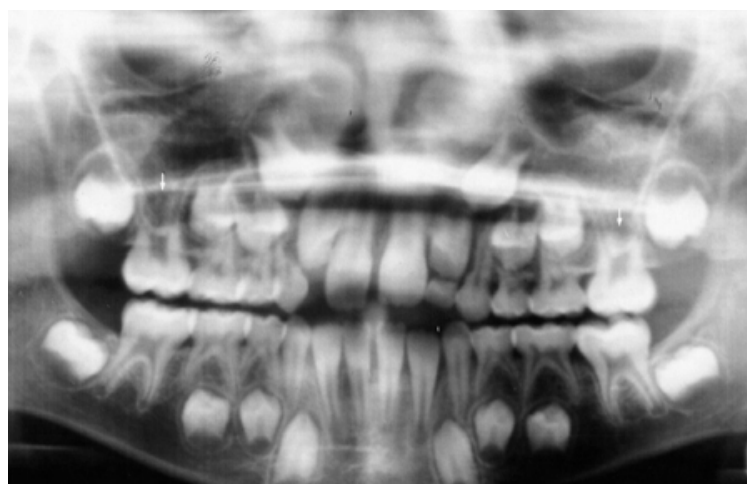

Figure 9 : Anomalies dentaires du patient M-C1 : racines grêles $(16,26)$

Dental anomalies of the patient M-C1: thinned roots $(16,26)$

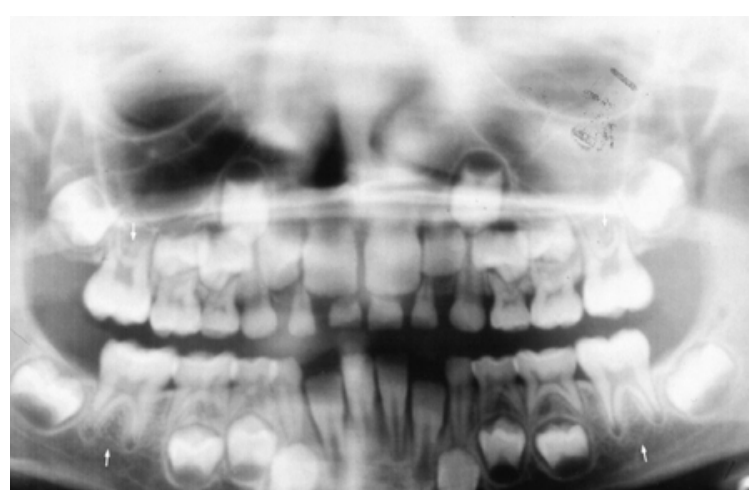

Figure 11 : Anomalies dentaires du patient M-C6 : racines grêles $(16,26,36,46)$

Dental anomalies of the patient M-C6: thinned roots $(16,26,36,46)$ 


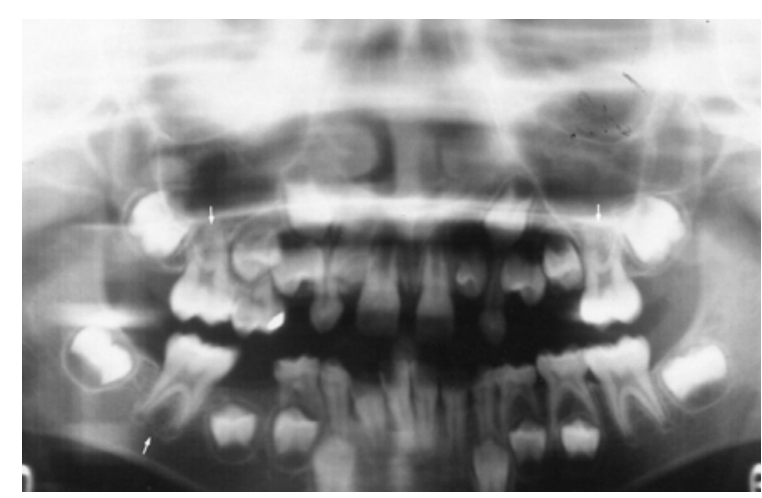

Figure 12 : Anomalies dentaires du patient $\mathrm{M}-\mathrm{C} 7$ : racines grêles $(16,26,46)$

Dental anomalies of the patient $M-C 7$ : thinned roots $(16,26,46)$

La moyenne d'âge de début de la chimiothérapie est de 5 ans. Un cas de microdontie est noté (Fig 6). Ici il concerne un patient dont l'âge d'administration de la chimiothérapie est inférieur à 3 ans; pour Maguire [10], $38 \%$ des enfants ayant reçu une chimiothérapie avant 3 ans présentent des microdonties.

Dans le groupe $T$, il existe une agénésie d'une seconde prémolaire mandibulaire, soit $9.09 \%$ à titre indicatif. Avec les réserves dues à l'effectif du groupe, ce chiffre se rapproche des $7.73 \%$ de la population générale cités par Monteil [27]. Remarquons que la seule agénésie de ce groupe atteint aussi la dent la plus fréquemment absente pour tous les auteurs [27-30]. C'est une dent de fin de série, mais ici l'agénésie est unilatérale contrairement aux patients malades. Cinq patients ne présentent aucune anomalie répertoriée et il est à noter que 3 d'entre eux étaient les plus âgés (en phase d'édification radiculaire de la 47 ) au moment des cures.

\section{Influence de la chimiothérapie sur le rythme d'édification de la 47}

\section{Section résultats - analyse statistique}

La comparaison des rythmes de minéralisation annuels coronaires et radiculaires révèle l'absence de différence statistiquement significative entre les groupes $M$ et $T$ (Tab. 4). Cependant, les données du groupe $M$ représentent des rythmes globaux, aussi il convient d'analyser

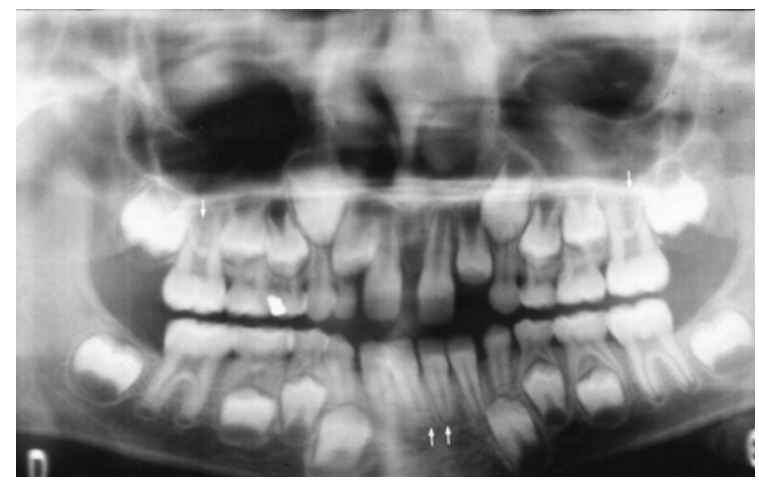

Figure 13 : Anomalies dentaires du patient M-C8 : racines courtes $(31,41)$, grêles $(16,26)$

Dental anomalies of the patient M-C8: shortening (31, $41)$ and thinned roots $(16,26)$

les données de ce groupe en y distinguant les valeurs observées d'une part pendant les périodes sous chimiothérapie et les périodes sans chimiothérapie, et d'autre part selon la phase de chimiothérapie: phase de chimiothérapie d'induction-consolidation (IC) et phase de chimiothérapie d'entretien (E).

La comparaison des accroissements coronaires et radiculaires entre les périodes avec ou sans chimiothérapie montre que l'édification coronaire de 47 est significativement ralentie pendant l'application de la chimiothérapie $(p=0,0109)$, en revanche il n'y a pas de différence significative pour l'édification radiculaire entre les périodes avec ou sans chimiothérapie (Tab. 5).

La comparaison de ces accroissements selon les phases de chimiothérapie $I C$ et $E$ révèle que la couronne de 47 a une croissance significativement plus rapide $(p=0,0473)$ pendant la phase $\mathrm{E}$ que pendant la phase IC et qu'il n'est constaté aucune différence significative pour la minéralisation radiculaire de 47 quelle que soit la phase de chimiothérapie (Tab. 6).

Enfin, la comparaison des accroissements coronaires lors des phases IC et E versus les périodes sans chimiothérapie montre que le rythme de calcification coronaire observé de 47 est très significativement moins rapide $(p=0,0033)$ pendant la chimiothérapie d'induction-consolidation qu'en l'absence de chimiothérapie alors qu'il n'existe aucune différence médecine

buccale chirurgie buccale

VOL. $9, \mathrm{~N}^{\circ} 1$ 2003

page 17 
Tableau 4 : Comparaison des accroissements coronaires et radiculaires de 47 entre les groupes $M$ et $T$ (mm.an ${ }^{-1} ; \mu \pm \mathrm{SD}$ ), test de Wilcoxon.

Comparison of crown mineralization rythm and root mineralization rythm of the mandibular second right molar between treated and control groups. (mm.year ${ }^{1}$; $\mu \pm S D)$, Wilcoxon test.

\begin{tabular}{|cccc|}
\hline Accroissement & Groupe M & Groupe T & p \\
Coronaire & $1,43 \pm 0,58$ & $1,62 \pm 0,35$ & NS \\
Radiculaire & $1,76 \pm 0,79$ & $1,60 \pm 0,57$ & NS \\
\hline
\end{tabular}

Tableau 5 : Comparaison des accroissements coronaires et radiculaires de 47 entre les périodes avec et sans chimiothérapie (mm.an $\left.{ }^{-1} ; \mu \pm S D\right)$, test de Wilcoxon.

Comparison of crown mineralization rythm and root mineralization rythm of the mandibular second right molar between periods with and without chemotherapy. (mm.year $\left.{ }^{1} ; \mu \pm S D\right)$, Wilcoxon test.

médecine buccale chirurgie buccale

VOL. $9, \mathrm{~N}^{\circ} 1$ 2003

page 18

\begin{tabular}{|cccc|}
\hline Accroissement & Avec chimio & Sans chimio & $\mathbf{p}$ \\
Coronaire & $1,27 \pm 0,50$ & $1,84 \pm 0,54$ & 0.0109 \\
Radiculaire & $1,52 \pm 0,74$ & $2,35 \pm 0,74$ & NS \\
\hline
\end{tabular}

Tableau 6 : Comparaison des accroissements coronaires et radiculaires de 47 entre les phases de chimiothérapie (mm.an $\left.{ }^{-1} ; \mu \pm \mathrm{SD}\right)$. Test de Kruskal et Wallis pour l'accroissement coronaire, test de Wilcoxon pour l'accroissement radiculaire.

Comparison of crown mineralization rythm and root mineralization rythm of the mandibular second right molar between phases of chemotherapy. (mm.year ${ }^{1}$; $\mu \pm S D)$, Kruskal and Wallis test for crown and Wilcoxon test for root.

\begin{tabular}{|cccc|}
\hline Accroissement & Chimio IC & Chimio E & $\mathbf{p}$ \\
Coronaire & $1,00 \pm 0,37$ & $1,51 \pm 0,52$ & 0,047 \\
Radiculaire & $1,15 \pm 0,41$ & $2,08 \pm 0,91$ & NS \\
\hline
\end{tabular}

significative entre la chimiothérapie d'entretien et l'absence de chimiothérapie (Tab. 7). La chimiothérapie d'induction-consolidation ralentit la minéralisation coronaire alors que le traitement d'entretien n'a pas d'effet statistiquement perceptible. La même comparaison pour les accroissements radiculaires ne montre pas de différence significative entre les périodes sous et sans chimiothérapie quelle que soit la phase du traitement (Tab. 7). Cependant, l'effectif statistique souhaitable n'étant pas atteint, ce résultat ne peut être validé.

\section{DISCUSSION}

L'absence chez les enfants malades de chirurgie, d'hormonothérapie de croissance et de radiothérapie élimine de fréquents facteurs de doute. Mais il n'est pas possible ici de différencier l'effet des antimitotiques de celui des corticoïdes ni d'identifier la ou les drogues responsables de ces troubles. Notons que des études expérimentales menées sur les germes des deuxièmes molaires du hamster semblent incriminer les antibiotiques antitumoraux, l'actinomycine $\mathrm{D}$ et la vincristine comme les drogues les plus cytotoxiques pour le système dentaire avec les effets les plus irréversibles [19]. Les résultats de cette étude convergent pour établir un ralentissement de la minéralisation coronaire par la chimiothérapie alors que l'édification radiculaire ne semble pas touchée. Les tests identifient l'induction-consolidation, phase intensive et toxique, comme phase responsable alors que les effets de la chimiothérapie d'entretien ne sont pas statistiquement perceptibles. Le ralentissement de la minéralisation coronaire semble indiquer une plus grande fragilité de la 47 dans la première partie de son développement et donc aux premiers âges ( $2^{1 / 2}$ à 8 ans).

Tableau 7 : Comparaison des accroissements coronaires et radiculaires de 47 entre les phases de chimiothérapie et sans chimiothérapie (mm.an $\left.{ }^{-1} ; \mu \pm \mathrm{SD}\right)$, test de Wilcoxon.

Comparison of crown mineralization rythm and root mineralization rythm of the mandibular second right molar between phases of chemotherapy and without chemotherapy. (mm.year $\left.{ }^{1} ; \mu \pm S D\right)$, Wilcoxon test.

\begin{tabular}{|cccccc|}
\hline Accroissement & Chimio IC & Chimio E & Sans chimio C- & p IC/C- & p E/C- \\
Coronaire & $1,00 \pm 0,37$ & $1,51 \pm 0,52$ & $1,84 \pm 0,54$ & 0,0033 & NS \\
Radiculaire & $1,15 \pm 0,41$ & $2,08 \pm 0,91$ & $2,35 \pm 0,74$ & NS & NS \\
\hline
\end{tabular}


Bien que le rythme de développement de la racine de la 47 ne semble pas influencé par les antimitotiques, il est observé à l'instar d'autres auteurs une proportion importante de malformations radiculaires. Cette contradiction n'est peut-être qu'apparente, on ne peut totalement exclure qu'une racine courte puisse s'être développée à la même vitesse qu'une racine normale mais sur un laps de temps plus bref. L'absence d'influence de la chimiothérapie d'entretien sur le rythme d'édification de la 47 n'exclut en rien son rôle dans l'apparition d'anomalies (coronaires ou radiculaires). Le nombre de cas étudiés en phase radiculaire limite la portée des conclusions.

Concernant les anomalies dentaires rencontrées, 8 enfants malades sur 13 ont présenté des troubles du développement dentaire corrélés à la chimiothérapie reçue en se référant aux tables de Schour et Massler [24]. Ce chiffre est sous-estimé par la non prise en compte possible pour le moment, d'éventuels défauts structurels amélo-dentinaires. Les anomalies les plus récurrentes sont les racines grêles et/ou courtes et les agénésies. La microdontie et les agénésies ont touché les enfants traités avant l'âge de 5 ans. Les agénésies sont multiples et bilatérales mais quelle que soit l'anomalie, la dent la plus fréquemment atteinte est la seconde prémolaire.

Cette étude doit être complétée par l'examen clinique de la denture des enfants suivis, à la recherche d'éventuels défauts amélo-dentinaires. Ces enfants nécessitent une surveillance dentaire approfondie et permanente. Si un traitement orthodontique devait être entrepris pour combler par exemple les espaces correspondant aux agénésies, il faut avoir à l'esprit que le parodonte a pu être éventuellement atteint. Si à l'avenir chez ces enfants des dents permanentes devaient être extraites, il serait intéressant de pouvoir pratiquer une analyse physico-chimique structurale. Les perspectives seraient aussi d'identifier les drogues les plus toxiques pour le système dentaire ainsi que les stades du développement cellulaire les plus sensibles aux antimitotiques.

effects of treatment with chemotherapy and radiation to the head and neck. Pediatrics 1984; 73: 816-23.

7 - KENRAD B. Et tilfaelde af tanddannelsesforstyrrelser efter cytostatikabehandling. Tandlaegebladet 1984; 19: 687-9.

8 - Welbury RR, Craft aW, Murray JJ, Kernahan J. Dental health of survivors of malignant disease. Arch Dis Child 1984; 59: 1186-7.

9 - Rosenberg SW, Kolodney H, Wong Gy, Murphy ML. Altered dental root development in long-term survivors of pediatric acute lymphoblastic leukemia: a review of 17 cases. Cancer 1987; 59: 1640-8.

10 - Maguire A, Craft aW, Evans RGB, Amineddine $H$, Kernahan J, Macleod RI, Murray JJ, Welburry RR. The long-term effects of treatment on the dental condition of children surviving malignant disease. Cancer 1987; 60: 2570-5.

11 - Nunn JH, Welbury RR, Gordon PH, Kernahan J, CRAFT AW. Dental caries and dental anomalies in children treated by chemotherapy for malignant disease: a study in the north of England. International J Paediatr Dent 1991; 1: 131-5. médecine buccale chirurgie buccale

VOL. $9, \mathrm{~N}^{\circ} 1$ 2003

page 19
5 - DURR DP, AdAIR SM, Novak EV. Dental abnormalities associated with the treatment of Hodgkin's disease in a young patient. J Pedod 1987; 12: 98-104.

6 - Jaffe N, Toth BB, Hoar RE, Reid HL, Sullivan MP, MACNEESE MD. Dental and maxillo-facial abnormalities in long-term survivors of childhood cancer, 
12 - Salagnac JM, Leguillou-Negrea A, Mechinaud F, MERCIER J. Incidence des chimiothérapies antimitotiques sur la dentition : à propos de 71 observations. Rev Stomatol Chir Maxillofac 1996; 4: 229-40.

13 - Sonis AL, Tarbell N, Valachovic RW, Gelber R, SCHWENn M, Sallan S. Dentofacial development in long-term survivors of acute lymphoblastic leukemia. Cancer 1990; 66: 2645-52.

14 - DAHLLÖF G, BARR M. Disturbances in dental development after total body irradiation in bone marrow transplant recipients. Oral Surg Oral Med Oral Pathol. 1988; 65: 41-6.

15 - DahllöF G, Rozell B, Forsberg CM, Borgstrom B. Histologic changes in dental morphology induced by high dose chemotherapy and total body irradiation. Oral Surg Oral Med Oral Pathol 1994; 77: 56-60.

16 - AdATIA AK. The effects of cyclophosphamide on odontogenesis in the rat. Arch Oral Biol 1975; 20: 141-4.

17 - Koppang HS. Effects of cyclophosphamide of dentinogenesis in the rat incisor : fluorescence, microscopic and microradiographic investigations. Scand $J$ Dent Res 1981; 89 : 59-70.

médecine buccale chirurgie buccale

VOL. $9, \mathrm{~N}^{\circ} 1$ 2003

page 20
21 - Cavézian R, Pasquet G. Imagerie et diagnostic en Odonto-Stomatologie (pp 13-15). Ed. Masson, Paris, 1989.

22 - Danhiez P, SalÄUn M, Chaud D. Les distorsions des images dentaires en orthopantomographie. Rev Stomatol Odontol du Nord 1972; 1: 21-7.

23 - Blocquel H, Justin J, Lafforgue P, Declerce P, BAUDIN M. Etude télécrânienne du rythme de maturation de la seconde molaire inférieure. Bull Group Int Rech Sc Stom et Odontol 1981; 24: 81-88.

24 - SCHOUR I, MASSLER M. The development of the human dentition. J Am Dent Anoc 1941; 28: 1135-60.

25 - PAJARI U, LANNING M. Developmental defects of teeth in survivors of childhood A.L.L. are related to the therapy and age at diagnosis. Med Pediatr Oncol 1995; 24: 310-4.

26 - PAJARI U, LANNIng M, LARMAS M. Prevalence and location of enamel opacities in children after anti-neoplastic therapy. Community Dent Oral Epidemiol 1988; 16: $222-6$

27 - MONTEIL M. Contribution à l'étude des agénésies dentaires. Orthod fr 1962; 33; 185.

28 - NeMETH T. A propos d'agénésies. Pédodentol fr 1982; 16: 247-53.

29 - Bertrand E. Les anomalies dentaires de taille et de forme : microdontie, macrodontie et formations doubles. J odontostomatol Pediatr 1997; 1: 55-60.

30 - Brook A. Dental abnormalities of number, form and size: their prevalence in british schoolchildren. J Int Assoc Dent Child $1975 ; 5:$ 37-53.

31 - ADATIA AK. Effects of cytotoxic chemotherapy on dental development. J Roy Soc Med 1987; 12: 784-5. guës (pp 805-825) In : L'hématologie de Bernard Dreyfus. Breton-Gorius J, Reyes F, Rochant H, Rosa J, Vernant JP. Ed. Flammarion médecinesciences, Paris, 1992. 